\title{
Low Potential Stable Glucose Detection at Dendrimers Modified Polyaniline Nanotubes
}

\author{
Alessandra Nogueira Santos, Demétrio Artur Werner Soares, \\ Alvaro Antonio Alencar de Queiroz* \\ Departamento de Física e Química, Universidade Federal de Itajubá - UNIFEI, \\ Av. BPS, 1303, 37500-903 Itajubá, MG, Brazil
}

Received: December 11, 2007; Revised: January 12, 2010

\begin{abstract}
The utilization of nanostructured materials for development of biosensors is a growing field in medical diagnostics. In this work a glucose biosensor based on bioactive polyglycerol (PGLD) and chitosan dendrimers (CHD) was developed. PGLD and CHD were bioconjugated with the enzyme glucose oxidase (GOx) to obtain dendrimers with glucose sensing properties. Polyaniline nanotubes (PANINT's) were used as electron mediator due to their high ability to promote electron-transfer reactions involving GOx. The PGLD-GOx and CHD-GOx were entrapped in PANINT's during template electrochemical polymerization of aniline. The prepared PGLDGOx/PANINT's and CHD-GOx/PANINT's biosensors exhibit a strong and stable amperometric response to glucose even at a low potential of $+100 \mathrm{mV}$. The based PGLD-GOx/PANINT's and CHD-GOx/PANINT's biosensors showed a good performance in glucose concentrations range in human blood. A comparison of the sensitivities to glucose showed that both biosensors have a linearity range between 0.02 and $10 \mathrm{mM}$, though PGLD-GOx/PANINT's is more sensitive (10.41 vs. $\left.7.04 \mathrm{nA} \cdot \mathrm{mM}^{-1}\right)$. The difference in the biosensor behavior and the high sensitivity of the PGLD-GOx/PANINT's may be due to the specific organization of GOx layer at surface of the modifier macromolecule PGLD and their distribution in PANINT's. The enzyme affinity for the substrate, $\mathrm{K}_{\mathrm{M}}^{\text {app }}$ remains quite good after GOx immobilization on PGLD and CHD dendrimers and entrapment of the bioconjugates in PANINT's.
\end{abstract}

Keywords: polyglycerol dendrimers, chitosan dendrimers, polyaniline nanotubes, glucose biosensor

\section{Introduction}

The present century is marked by the nanotechnology where researchers have developed this technology to observe and manipulate atoms directly as result of the advances from microscopy, materials science, top-down and bottom-up synthesis and the relationship between classical and quantum physics ${ }^{1}$.

Today, dendrimers represents the most important and largest family of nanochemistry being used in medical technology ${ }^{2}$. The intrinsic properties of dendrimers like as monodispersity, highly functional groups at macromolecule periphery and good biocompatible properties of these nano-objects has led to their widespread use in a variety of applications in medicine and biotechnology ${ }^{3}$.

Recently, poly (amidoamine) (PAMAM) dendrimers have been synthesized with a variety of core materials, branching units and surface modifications. PAMAM dendrimers and their hybrid nanocomposites has been used with great success for the construction of highly sensitive and stable amperometric glucose biosensor demonstrating the high potential of these nano-particles as platform for the design of clinical biosensors for glucose monitoring ${ }^{4}$. However, while development of PAMAM dendrimer has tremendous technological potential, these materials, have been shown to exhibit significantly toxicity to mammalian cells ${ }^{5}$.

Recently, the good biocompatibility properties of polyglycerol (PGLD) and chitosan (ChD) dendrimers have received our attention for applications in medicine ${ }^{6-7}$. PGLD and CHD have shown to be a good matrix for the enzyme immobilization. Enzyme immobilization onto electrode surface is a crucial step in fabrication of amperometric bosensors.

In this work the enzyme glucose oxidase (GOx) was immobilized onto polyglycerol (PGLD) and chitosan dendrimers (CHD) (Figure 1). GOx has been chosen as a model enzyme in this study to explore the applicability of the PGLD and CHD as a matrix for enzymes of clinical interests. Glucose oxidase (GOx) is widely used in biosensors design for the estimation of the metabolite glucose in whole blood. The estimation of metabolites such as glucose in whole blood is of central importance in the modern therapy for diabetes mellitus ${ }^{8}$.

Glucose oxidase ( $\beta$-D-glucose:oxygen 1-oxidoreductase, EC1.1.3.4) is an enzyme that catalyses the oxidation of $\beta$-D-glucose to D-glucono-1,5-lactone and hydrogen peroxide, using molecular oxygen as the electron acceptor in according to the reaction:(Chart 1)

The generated hydrogen peroxide may be detected amperometrically at the enzyme electrode making use of mediators to shuttle electrons from the enzyme to the electrode surface.

The usefulness of CHD and PGLD dendrimers as the support for binding the GOx can be ascribed to: i) high functionality at macromolecule periphery that facilitates the enzyme immobilization procedure, ii) low cost and iii) good biocompatibility properties. The similarity in length scales and supramolecular structure between PGLD/CHD dendrimers and enzymes suggests interactions at GOx-dendrimer interface that may be favorable for biosensor electrode applications. Additionally, the bioconjugated PGLD-GOx and CHD-GOx until this 




(a)

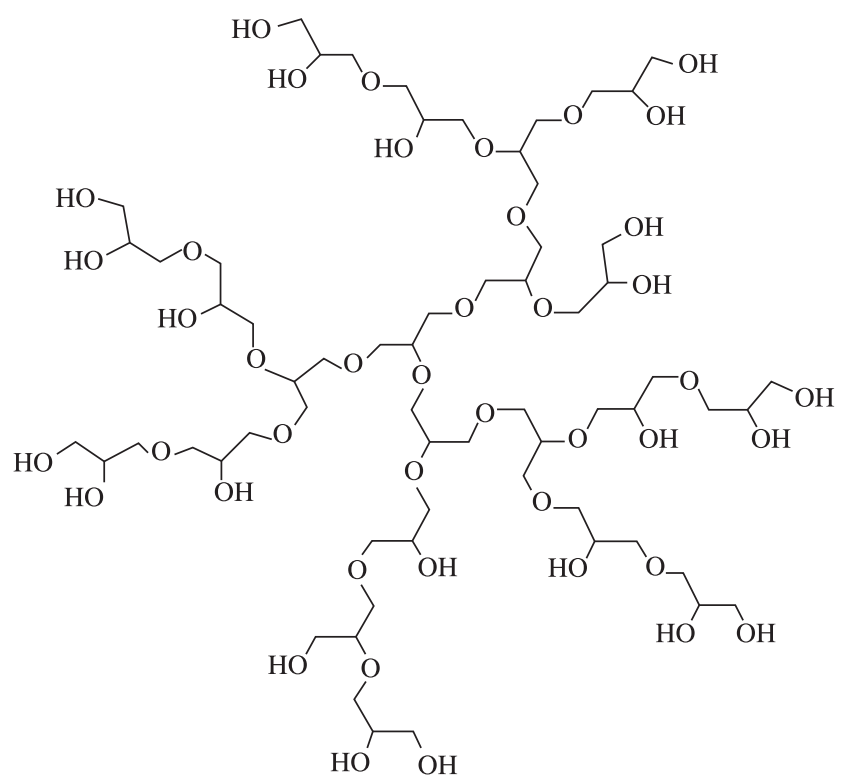

(b)

Figure 1. Schematic architectures of the CHD (a) and PGLD (b) dendrimers synthesized in this work for GOx immobilization.

moment were not well characterized electrochemically in literature as glucose biosensor, offering new directions in biosensor development. Enzyme entrapment, electron mediation and nano-biosensor fabrication are some of the possibilities that PGLD and CHD offer to the biosensing devices.

The electrical communication between redox centers of GOx and surface electrode was established through poly(aniline) nanotubes (PANINT's) for the transport of electrical charge. This approach has received particular attention for the construction of third generation glucose biosensors ${ }^{8}$. PANINT's possess many unique properties such as high electrocatalytic effect, fast electron transfer, environmental stability and ability to promote fast electron-transfer reactions involving the enzyme GOx9.

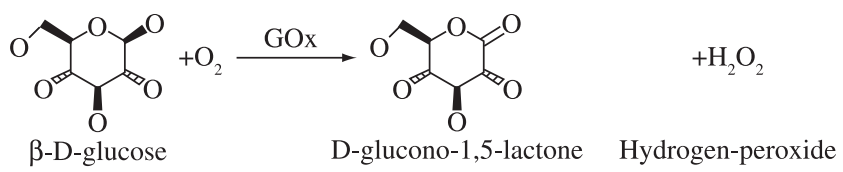

Chart 1 . The enzymatic reaction catalysed by glucose oxidase (GOx).

The kinetic parameters of PGLD-GOx/PANINT's and CHDGOx/PANINT's such as response time, sensitivity, detection limit, linear range and enzyme activity were determined and MichaelisMenten kinetics parameters were also evaluated. The behaviors of the PGLD-GOx and CHD-GOx entrapped in PANINT's matrix were also studied and compared with that of the free PGLD-GOx and CHDGOx. Such information is believed to be of practical importance for the rational design of the dendrimers as carriers of biomacromolecules in high-throughput clinical assays.

\section{Experimental}

\subsection{Dendrimer synthesis}

Detailed procedures for the synthesis and characterization of CHD and PGLD dendrimers are reported in other publications originated from our researcher group ${ }^{6-7}$. Briefly, the dendronized chitosan (CHD) was synthesized by repeating the Michael addition of methacrylic acid (10 mM, Sigma-Aldrich) to amino groups of chitosan (100 mM, Sigma-Aldrich) followed by the amidation of terminal ester groups by ethylene diamine $(2 \mathrm{mM})$. The dendronized chitosan (CHD) was purified by precipitation in methanol at room temperature $\left(25^{\circ} \mathrm{C}\right)$. The fourth generation polyglycerol dendrimer (PGLD) was prepared by the slow addition of glycidol to a solution containing a partially deprotonated polyol-core at $120^{\circ} \mathrm{C}$ in argon atmosphere for 24 hours. Both, CHD and PGLD dendrimers were dialyzed and lyophilized before enzyme immobilization.

\subsection{Covalent immobilization of glucose oxidase on the CHD and PGLD dendrimers}

The enzyme glucose oxidase (GOx E.C.1.1.3.4, 148 U.mg $^{-1}$, Sigma-Aldrich) was chemically immobilized onto CHD and PGLD dendrimer surfaces through a condensation reaction using activation of the - $\mathrm{OH}$ (PGLD) or - $\mathrm{NH}_{2}$ (CHD) dendrimers groups by a cyanylating reaction to the hydroxyl groups on dendrimer surface via amide bond using 1-Cyano-4-(dimethylamino)-pyridinium tetrafluoroborate $(\mathrm{CDAP})^{10}$. Leaving the activated CHD and PGLD dendrimers in the GOx solutions ( $3 \mathrm{mg} \cdot \mathrm{mL}^{-1}$ ) at $4{ }^{\circ} \mathrm{C}$ overnight completed the enzyme immobilization. The bioconjugates CHD-GOx and PGLD-GOx were purified by dialysis and lyophilized. The amount of immobilized GOx was calculated by measuring the concentration of the protein in the supernatant and then subtracting from the total free enzyme amount using the classic Bradford methodology ${ }^{11}$.

\subsection{Preparation of enzyme electrode}

The glucose biosensing electrodes based on the CHD-GOx and PGLD-GOx was prepared by immobilizing the bioconjugates on protonated polyaniline nanotubes (PANINT's) polarographicaly deposited at thin aluminum films (Alcoa, 99.99\%, 30.0 $\mu \mathrm{m}, 2 \mathrm{~V}$ ) in solution containing aniline (Sigma-Aldrich, 99.0\%, $2.2 \mathrm{mM}$ ) and $\mathrm{H}_{2} \mathrm{SO}_{4}$ (Merck, $\left.1 \mathrm{M}\right)$ at room temperature $\left(27{ }^{\circ} \mathrm{C}\right)$ in according to our recent works ${ }^{12}$.

Electrochemical polymerization solution consisted of redistilled aniline $(0.1 \mathrm{M})$, the bionjugated dendrimer $\left(4.2 \mathrm{U} \cdot \mathrm{mL}^{-1}\right)$ and p-toluene sulphonate $(1 \mathrm{M})$ in sodium phosphate buffer saline (NaPBS) $(0.1 \mathrm{M}$, $\mathrm{NaH}_{2} \mathrm{PO}_{4} / \mathrm{Na}_{2} \mathrm{HPO}_{4}, 0.05$ mol.L $\mathrm{L}^{-1} \mathrm{NaCl}, \mathrm{pH}$ 7.4) as electrolyte. The 
nanotubes growth was terminated in the potential cycle at $-100 \mathrm{mV}$ vs. a silver calomelan electrode $(\mathrm{Ag} / \mathrm{AgCl}, \mathrm{SCE})$, so that the PANINT's were in the reduced state and to achieve an estimated charge loading of $30 \mathrm{mC}$. The electrodes were thoroughly washed with NaPBS and stored in the buffer solution at room temperature $\left(25^{\circ} \mathrm{C}\right)$ for 24 hours to remove any excess on residual monomer and GOx weakly bound to the PANINT's. After the electrochemical entrapment of the PGLD-GOx and CHD-GOx within PANINT's, the bioactivities of the electrodes were measured amperometrically.

\subsection{Characterization of the enzyme electrode}

The morphologies of the gold-coated PGLD-GOx/PANINT's and CHD-GOx/PANINT's biosensors surfaces were analyzed using scanning electron microscopy (SEM). SEM measurements were conducted on a Phillips XL30 instrument using an accelerating voltage of $20 \mathrm{kV}$.

The sensitivity of the biosensor to glucose was assayed by measuring the current response. The principle of the determination of the current response is based on the formation of hydrogen peroxide during the GOx-catalyzed reaction, which is as follows: (Equation 1)

Glucose $+\mathrm{O}_{2}+\mathrm{H}_{2} \mathrm{O} \underline{\mathrm{GOx}}$ Gluconic acid $+\mathrm{H}_{2} \mathrm{O}_{2}$.

The $\mathrm{H}_{2} \mathrm{O}_{2}$ production may be followed using a platinum working electrode (anode) generally polarized at $+700 \mathrm{mV}$ against an $\mathrm{Ag} / \mathrm{AgCl}$ (SCE) reference electrode. At this potential the hydrogen peroxide was oxidized according to the reaction: (Equation 2)

$$
\mathrm{H}_{2} \mathrm{O}_{2} \rightarrow \mathrm{O}_{2}+2 \mathrm{H}^{+}+2 \mathrm{e}^{-}
$$

The electric current produced is proportional to the glucose concentration $^{13}$

The cell used to determine the response current consisted of PANINT's/CHD-GOx or PANINT's/PGLD-GOx, a platinum electrode, and a SCE electrode in NaPBS buffer. The background current, $\mathrm{I}_{b}$, of the enzyme electrode, which was allowed to decay to a steady state, was first determined at a given potential, $37^{\circ} \mathrm{C}$ and $\mathrm{pH}$ value of 7.4. Then, the enzyme electrode was moved immediately into a separate NaPBS containing a known concentration of glucose to measure the current response. The latter determination was carried out at the same potential, $37^{\circ} \mathrm{C}$ and $\mathrm{pH}$ of 7.4, as those of determination of the background current. The steady state current $I_{S}$ for the determination of glucose on the currenttime curve was taken as the measured value. Therefore, the response current I of the enzyme electrode is equal to the difference between the measured current $\mathrm{I}_{\mathrm{b}}$ and $\mathrm{I}_{\mathrm{s}}$, i.e., $\mathrm{I}=\mathrm{I}_{\mathrm{S}}-\mathrm{I}_{\mathrm{b}}$. This procedure was important to eliminate the effects of potential, $\mathrm{pH}$ and temperature on the PANINT's conductivity between two current measurements ${ }^{14}$.

The measurements of the current flow across the biosensors were performed with a programmable electrometer (Keithley ${ }^{\circledR}$ model 237). The working electrodes were the PGLD-GOx/PANINT's or CHDGOx/PANINT's, while the SCE electrode was used as reference.

\subsection{Statistical analysis}

The repeatability of the biosensors responses were investigated under each optimized experimental conditions. In each series of five electrodes prepared, the relative standard deviations were obtained as the mean of five independent measurements for the current response of both, PGLD-GOx/PANINT's and CHD-GOx/PANINT's biosensors.

\section{Results and Discussion}

Since the carbon nanotubes synthesis at past century, the studies of this one-dimensional nanomaterial have provided a fruitful ground of many exciting applications in clinical chemistry ${ }^{15}$. Current clinical research promises new ways to diagnose disease, to deliver specific therapy, and to monitor the effects acutely and non-invasively ${ }^{16}$.

Figure 2 shows the SEM micrograph of the PGLD-GOx/ PANINT's deposited at Al electrodes. The SEM microstructure analysis demonstrates the nanotubes formation obtained as a nucleation of aniline molecules into the formed aluminum pores when the oxidation process takes place. The mechanism of the aniline polymerization appears to proceeds with increment of new aniline molecules that can interact with each other, through the amine and iminium nitrogen interaction forming at the same time fibrous polyaniline nanostructures (PANINT's). In this sense, electrodes with glucose sensing properties were done by entrapment of the bioconjugates PGLD-GOx or CHD-GOx during the polyaniline nanotubes (PANINT's) growth on metallic surface.

The dendrimer topology is an important molecular parameter that determines their physical properties and applications. To control dendrimer topology and accurately architecture is currently a central theme in polymer science with the aim to prepare macromolecules with new properties ${ }^{17}$.

The main problem to solve in the immobilization of enzymes to biosensors fabrication concerns the support selection. In this sense, a number of criteria such as microbial resistance, thermal stability, chemical inertia and functional groups on the surface of the support must be considered in the use of dendrimers for the development of biosensors $^{18}$.

In this work it was studied the properties of the glucose biosensors that depend on the dendrimer topology. The basic parameters that are highly important in characterizing the properties of the biosensors are response time, sensitivity, linear range and Michaelis-Menten constant $\left(\mathrm{K}_{\mathrm{M}}\right)$.

The detection of glucose was performed amperometrically, based on measurement of the current of electrons flowing from bioconjugated dendrimer and PANINT's due to anodic degradation of hydrogen peroxide ${ }^{19}$.

The change in the response current of the PGLD and CHD biosensors with the potential are shown in Figure 3. The response current increases from 100 to $300 \mathrm{mV}$, and then increases more slowly with further increasing potential. The maximum current occurs at $400 \mathrm{mV}$ for both PGLD-GOx and CHD-GOx biosensors, respectively.

The current-time relationships when the potential of the PGLDGOx and CHD-GOx biosensors at $300 \mathrm{mV}$ are shown in Figure 4. It was found that the response current of the enzyme electrode easily reaches to steady state. From the results in Figure 4 the current saturation peak for PGLD-GOx biosensor is meaningfully higher than

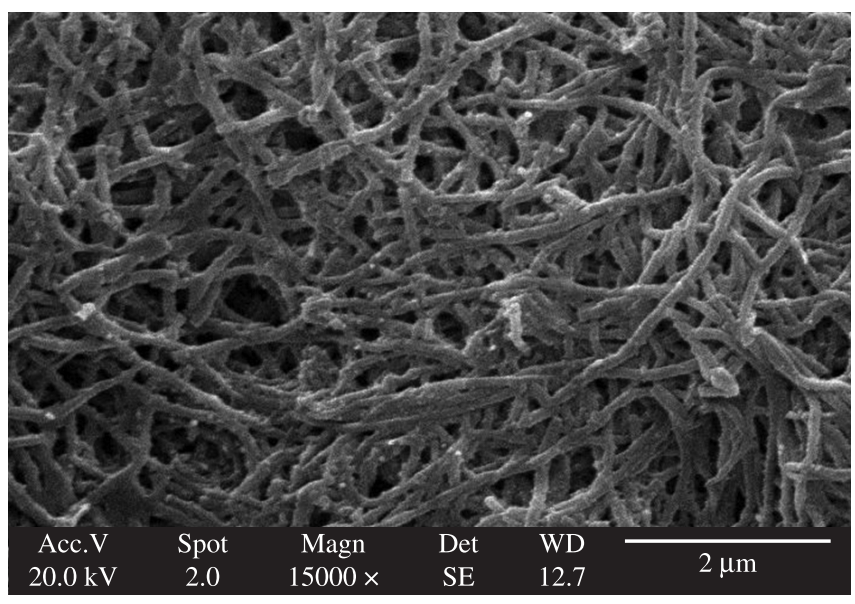

Figure 2. Surface SEM micrograph of the nanostructured PANINT's based PGLD and CHD glucose biosensor prepared in this work. 


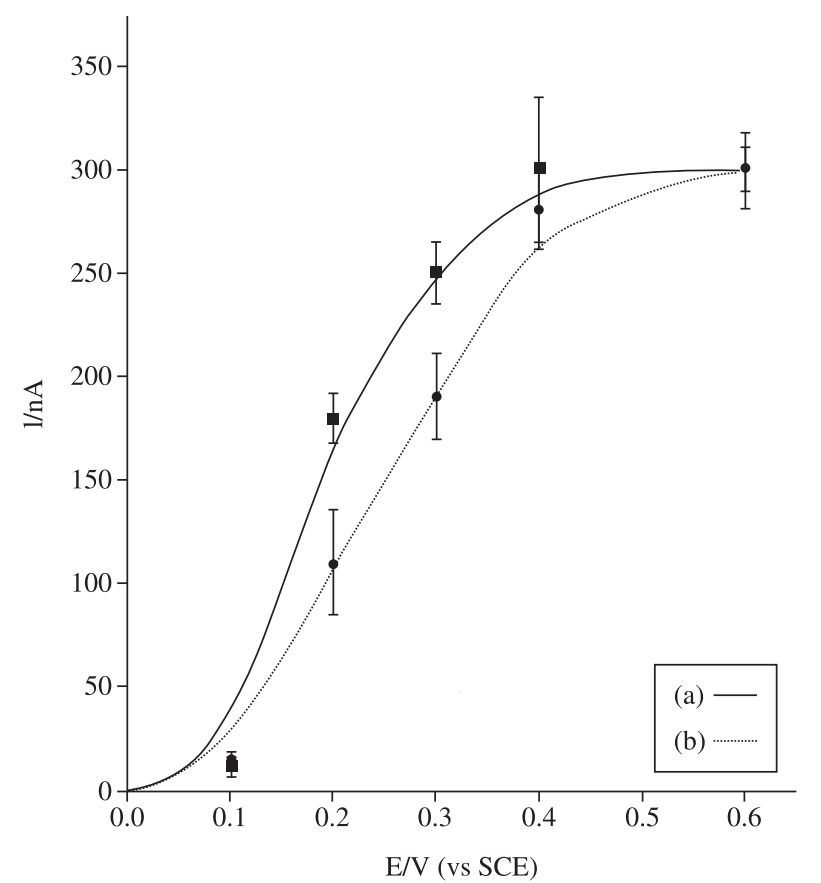

Figure 3. The relationship between the response current and the potential of the PGLD/GOx (a) and CHD/GOx (b) biosensors in NaPBS pH 7.4 at $37^{\circ} \mathrm{C}$. Glucose concentration: $20 \mathrm{mM}$. The error bars represent standard deviations between five electrodes.

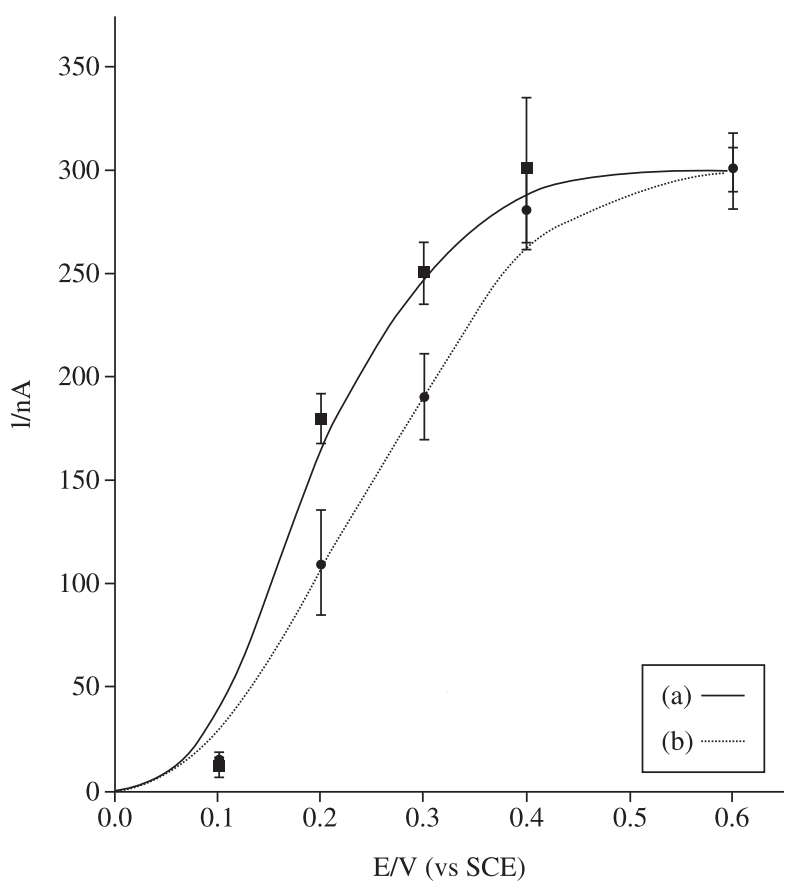

Figure 4. Current-time curve during the PGLD (a) and CHD (b) biosensors at $300 \mathrm{mV}$, pH 7.0 (NaPBS) and $37^{\circ} \mathrm{C}$ in glucose solution of $20 \mathrm{mM}$. The error bars represent the standard deviations between five electrodes.

CHD-GOx. The decay in response current observed in CHD-GOx biosensor may be due to the inhibition of GOx activity by the amino groups at CHD periphery. The periphery of CHD dendritic unit is filled with primary amine groups, while all the branching points, in the interior of the dendrimer, are occupied by tertiary amine. It is possible that the primary amino functions present in the periphery of

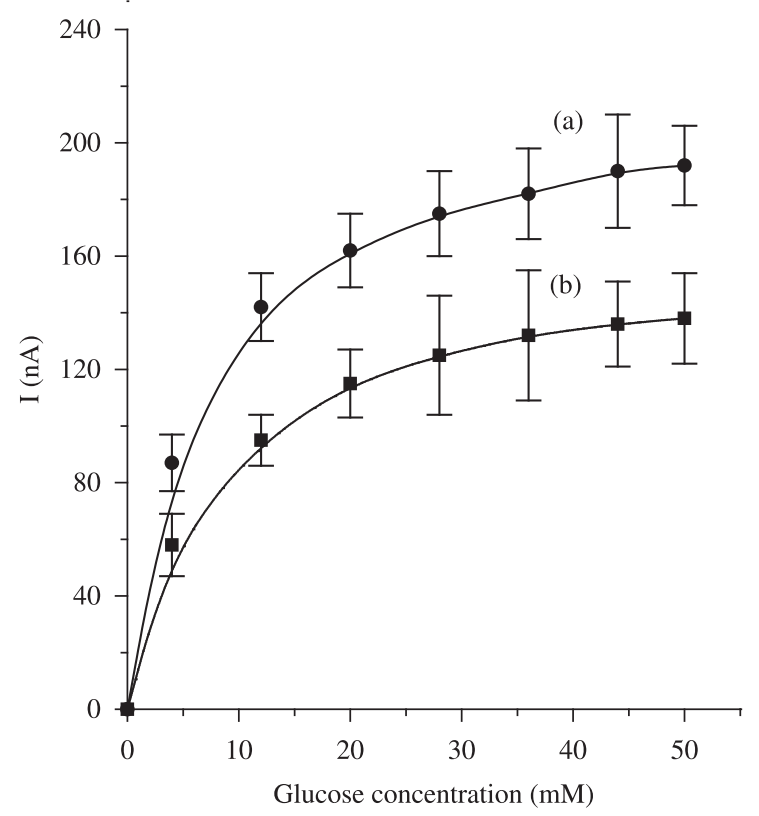

Figure 5. The relationship between response current and glucose concentration for the PGLD (a) and CHD (b) biosensors in $0.1 \mathrm{M} \mathrm{NaPBS}$ and $\mathrm{pH} 7.0$ at $37^{\circ} \mathrm{C}$. The error bars represent standard deviations between five electrodes.

the dendritic units may also participate through hydrogen bonds with the catalytic site of GOx resulting in a decrease of the bioactivity of the CHD biosensor.

The relationship between response current and glucose concentration in NaPBS buffer is shown in Figure 5. A characteristic hyperbolic Michaelis-Menten kinetics mechanism may be observed. It was found that, current increases with increasing glucose concentration in the range 1-50 $\mathrm{mM}$. Considering that the PGLD-GOx and CHD-GOx are uniformly distributed throughout the PANINT's, the reaction appears to be predominantly in the surface of the biosensors in the lower glucose concentration. However, the reaction on the surface of the electrodes and the diffusion occurring simultaneously at higher concentrations delays the response time. Thus, the increased glucose concentrations also increased the response current and finally reached to steady state value.

The evaluations of the PGLD-GOx/PANINT's and CHD-GOx/ PANINT's kinetics were determined by the analysis of the MichaelisMenten equation. The apparent Michaelis-Menten constant $\left(\mathrm{K}_{\mathrm{M}}{ }^{\text {app }}\right)$, which gives an indication of the enzyme-substrate kinetics can be calculated from the linearized electrochemical Michaelis-Menten equation form, denominated Lineweaver-Burk Equation (3) ${ }^{[20]}$ :

$$
\frac{1}{i_{S S}}=\frac{K_{M}^{a p p}}{i_{\text {Max }}} \frac{1}{[S]}+\frac{1}{i_{\text {Max }}}
$$

The parameter $\mathrm{K}_{\mathrm{M}}^{\text {app }}$ describes the enzymatic activity of GOx. A smaller $\mathrm{K}_{\mathrm{M}}{ }^{\text {app }}$ value indicates a more efficient GOx reaction. The $\mathrm{K}_{\mathrm{M}}{ }^{\text {app }}$ is related to the concentration of the substrate reaching the maximum rate of the enzyme-catalyzed reaction. Thus, the smaller $\mathrm{K}_{\mathrm{M}}{ }^{\text {app }}$ value, the lower is the substrate concentration for reaching the maximum value of the enzyme-catalyzed reaction. In this study, the $\mathrm{K}_{\mathrm{M}}{ }^{\text {app }}$ values for the PGLD-GOx and CHD-GOx were determined by using Lineweaver-Burk plot of the measured current-concentration data.

The relationship between $\mathrm{I}^{-1}$ against [glucose $^{-1}$ in $0.1 \mathrm{M}$ NaPBS $\mathrm{pH} 7.4$ for the PGLD-GOx and CHD-GOx dendrimers are shown in Figure 6. The maximum current response $\mathrm{I}_{\mathrm{Max}}$ and the apparent 


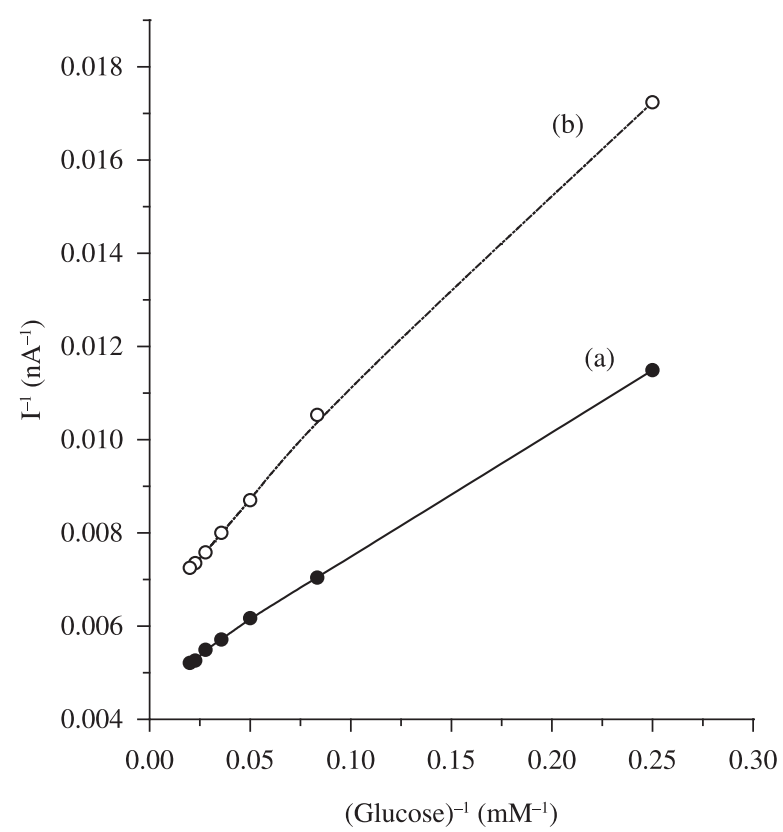

Figure 6. Electrochemical Lineweaver-Burk plot for PGLD (a) and CHD (b) biosensors based on the data of Figure 5 .

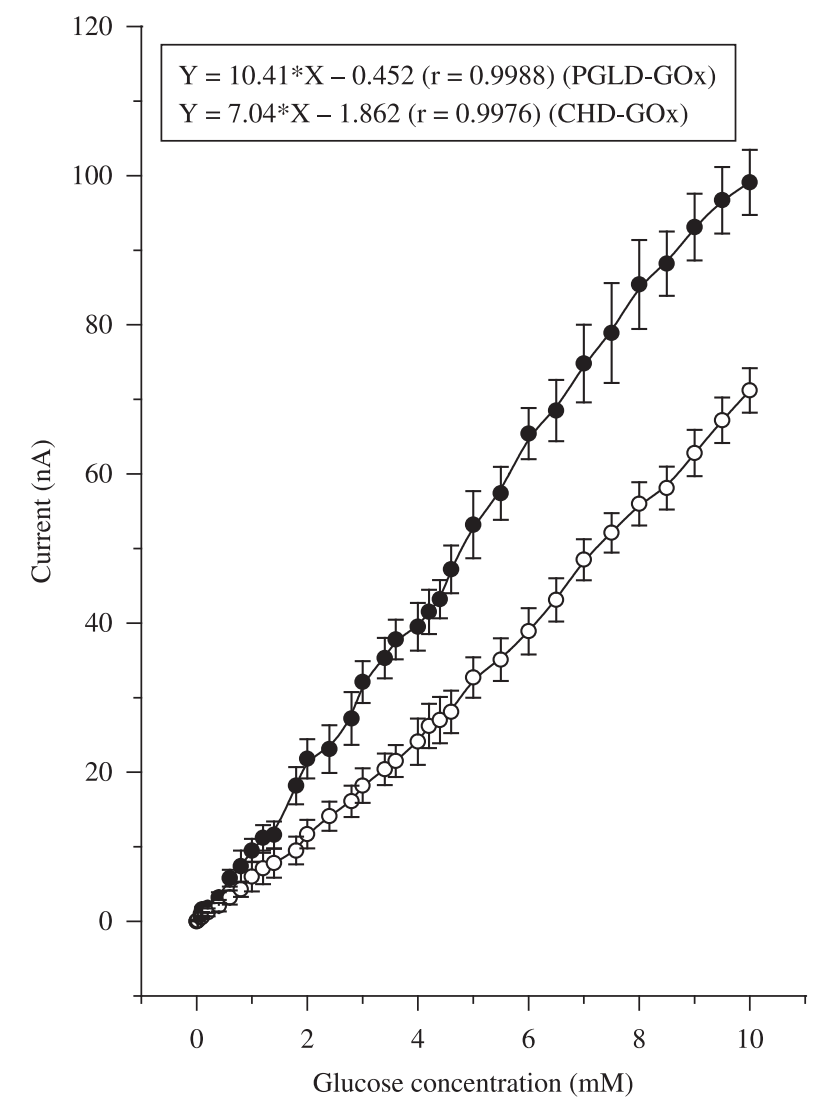

Figure 7. The calibration curve for glucose at the PGLD-GOx/PANINT's $(\bullet)$ and CHD-GOx/PANINT's ( $\left(\right.$ ) in NaPBS $0.1 \mathrm{M}, \mathrm{pH} 7.4,25^{\circ} \mathrm{C}$; applied potential, $300 \mathrm{mV}$. Error bars: \pm S.D., $\mathrm{n}=5$.

Michaelis-Menten constant $\mathrm{K}_{\mathrm{M}}{ }^{\text {app }}$ were calculated from the intercept and slope of the straight line. The maximum current responses for the bioconjugates CHD-GOx and PGLD-GOx immobilized in PANINT's were 0.154 and $0.211 \mu \mathrm{A}$, respectively. The $\mathrm{K}_{\mathrm{M}}{ }^{\text {app }}$ were 6.71 and
$5.75 \mathrm{mM}$ for the biosensors based on CHD-GOx and PGLD-GOx, respectively. The kinetic parameters, $\mathrm{K}_{\mathrm{M}}$ app of the free bioconjugates PGLD-GOx and CHD-GOx were $7.5 \pm 0.82 \mathrm{mM}$ and $6.3 \pm 0.58 \mathrm{mM}$ at constant temperature $\left(37^{\circ} \mathrm{C}\right)$ and $\mathrm{pH} 7.4$ (NaPBS), respectively. The PGLD-GOx and CHD-GOx entrapped in PANINT's exhibited lower $\mathrm{K}_{\mathrm{M}}^{\text {app }}$ values that of the free bioconjugates. The smaller $\mathrm{K}_{\mathrm{M}}$ app exhibited by the CHD-GOx and PGLD-GOx entrapped in PANINT's indicate that the electron mediator matrix did not cause any significant hindrance of the GOx towards its substrate.

For the fabrication of biosensors, different materials and methods of immobilization of enzymes have been used and these could result in different conformational changes in the enzyme structures given that the enzyme kinetics is environment sensitive. However, it is very difficult to compare one biosensor to others because the performance of the biosensor is greatly dependent on the applied potential, GOx immobilization method, the electrode material and its surface area. The $\mathrm{K}_{\mathrm{M}}{ }^{\text {app }}$ of PGLD-GOx and CHD-GOx were not affected rather prominently by the entrapment of bioconjugates in PANINT's and the values obtained for the Michaelis-Menten constant of the enzymes electrodes in this work are comparable to most of glucose biosensors based on polyaniline nanofibers ${ }^{21}$. Additionally, the similarity in $\mathrm{K}_{\mathrm{M}}$ app for PGLD-GOx and CHD-GOx indicate that both biosensors, PGLD-GOx/PANINT's and CHD-GOX/PANINT's are operating in enzyme kinetic controlled mode since the diffusion limitations in this case appear to be minimal22.

Figure 7 shows the calibration curves of the PGLD-GOx/ PANINT's and CHD-GOx/PANINT's. The linear range scans the glucose concentration from 0.02 to $10 \mathrm{mM}$. The calibration curves were linear with glucose concentration and sensitivities of 10.41 nA.mM ${ }^{-1}$ for PGLD-GOx/PANINT's and 7.04 nA.mM ${ }^{-1}$ for CHD-GOx/PANINT's were obtained. The results showed that the sensitivity of the PGLD-GOx/PANINT's biosensor to glucose $\left(10.41\right.$ nA.mM $\left.{ }^{-1}\right)$ is 1.5 larger than that of the CHD-GOx/PANINT's biosensor at working potential of $300 \mathrm{mV}$. This indicated that the structure of PGLD-GOx makes oxygen and glucose more easily accessible to the active site of enzyme than CHD-GOx structure. The sensitivity of PGLD-GOx/PANINT's and CHD-GOx/PANINT's appears to be comparable to sensitivity of those glucose biosensors that make use nanostructured polyaniline as electron mediator ${ }^{21-23}$.

The stability of glucose biosensors is also a very important factor, which influence the application and detecting capacity of the biosensor. In order to study the stability and reproductively of the PGLD-GOx/PANINT's and CHD-GOx/PANINT's biosensors were tested in the presence of $2 \mathrm{mM}$ glucose solution for 10 days (Figure 8). The biosensors were removed from a $4{ }^{\circ} \mathrm{C}$ refrigerator and put in the reaction cell to perform the experiment each day, and then dried and stored at $4{ }^{\circ} \mathrm{C}$ again after each experiment was completed.

Figure 8 shows the comparison of the stability of the PGLD-GOx/ PANINT's and the CHD-GOx/PANINT's glucose biosensors. In this work, the activity remained percentage was defined as the ratio of the activity remained after using a period of time to the initial activity, which is the quantitive expression of the stability. The response of the PGLD-GOx/PANINT's glucose biosensor remains stable throughout the entire experiment, with more than $70 \%$ of its initial activity after continuously using. In contrast, the CHD-GOx/PANINT's glucose biosensor displays a rapid decay of the response, with almost losing $11 \%$ of its activity after continuously using only about one day, which indicates an inhibition of the oxidation process capacity of GOx. This result may be explained by the integration of PGLD-GOx with PANINT's. The bioconjugate PGLD-GOx can be dispersed more uniformly in the PANINT's than CHD-GOx. In this case, PGLD-GOx can exert its catalytic property more adequately. 


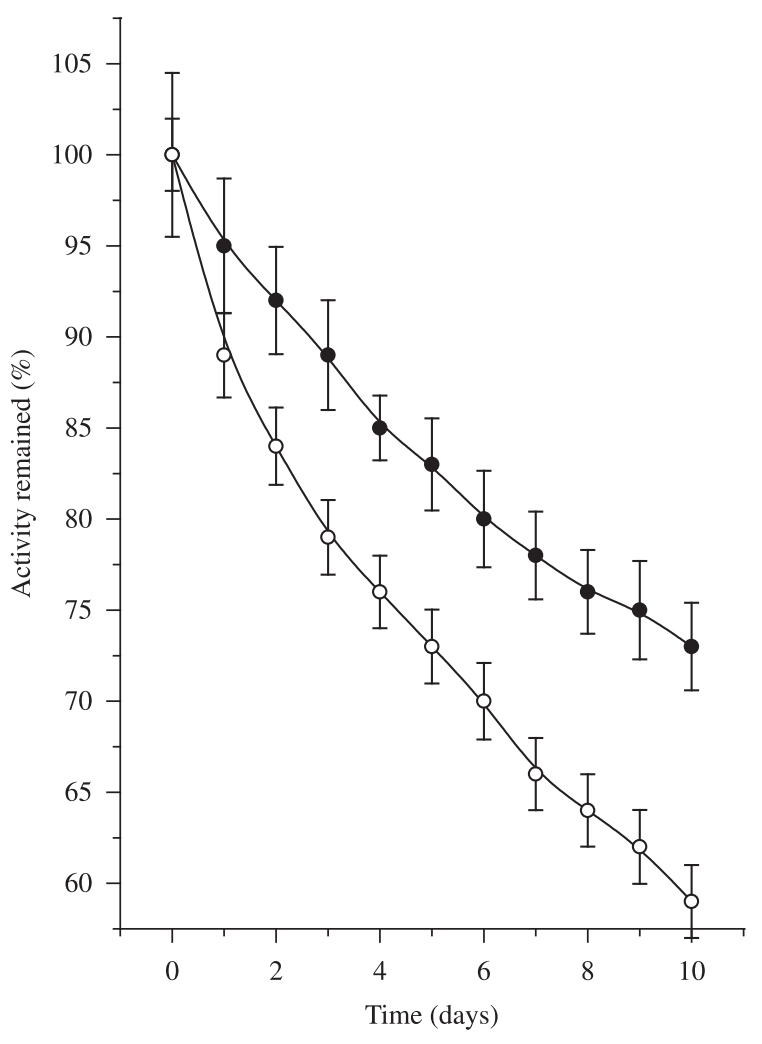

Figure 8. Stability of PGLD-GOx/PANINT's $(\bullet)$ and CHD-GOx/PANINTs (०) glucose biosensors at NaPBS $0.1 \mathrm{M}, \mathrm{pH} 7.4$ and $25^{\circ} \mathrm{C}$. The error bars represent standard deviations between five electrodes.

\section{Conclusions}

This study showed that PGLD and CHD dendrimers could be used successfully as nanoplatforms for the immobilization of GOx. PANINT's can be efficiently used as electron mediators in PGLDGOx and CHD-GOx biosensors. The measurements of MichaelisMenten kinetics indicated that PGLD-GOx/PANINT's has a better performance than CHD-GOx/PANINT's probably due to the more uniformly dispersion of PGLD-GOx in the PANINT's. However, both PGLD-GOx/PANINT's and CHD-GOx/PANINT's biosensors exhibits improved analytical performance with respect to their glucose sensing properties.

The use of the PGLD-GOx and CHD-GOx for construction of glucose biosensors may facilitate the fabrication of biosensors at nanoscale, promoting the integration of labile biological components into high throughput testing instruments and at same time biocompatible biosensors for implantation. These glucose biosensor materials are expected to have superior biocompatibility, which can be revealed by further studies.

\section{Acknowledgements}

This work was supported in part by a grant from the Finep, CNPq, Capes and Fapemig.

\section{References}

1. Baird D, Nordmann A and Schummer J, editors. Discovering the Nanoscale. Amsterdam: IOS Press; 2004. p. 145-156.

2. Tomalia DA. Birth of a new macromolecular architecture: dendrimers as quantized building blocks for nanoscale synthetic organic chemistry. Progress in Polymer Science. 2005; 30(3-4): 294-324.
3. Esfand R and Tomalia DA. Poly(amidoamine) (PAMAM) dendrimers: from biomimicry to drug delivery and biomedical applications. Drug Discovery Today. 2001; 6:427-436.

4. Svobodová L, Snejdárková M and Hianik T. Properties of glucose biosensors based on dendrimer layers. Effect of enzyme immobilization. Analytical and Bioanalytical Chemistry. 2002; 373(8):735-741.

5. Roberts JC, Bhalgat MK and Zera RT. Preliminary biological evaluation of polyamidoamine (PAMAM) starburst dendrimers. Journal of Biomedical Materials Research. 1996; 30(1):53-65.

6. Fernandes EGR, De Queiroz AAA, Abraham GA and San Romàn J. Antithrombogenic properties of bioconjugate streptokinase-polyglycerol dendrimers. Journal of Materials Science: Materials in Medicine. 2006; 17(2):105-111.

7. De Queiroz AAA, Abraham GA, Camillo MAP, Higa OZ, Silva GS, Del Mar Fernández M. et al. Physicochemical and antimicrobial properties of boroncomplexed polyglycerol-chitosan dendrimers. Journal of Biomaterials Science: Polymer Edition. 2006; 17(6): 689-707.

8. Renard E. Monitoring glycemic control: the importance of selfmonitoring of blood glucose. American Journal of Medicine. 2005; 118(suppl 9A):12S-19S.

9. Cosnier S. Biomolecule immobilization on electrode surfaces by entrapment for attachment to electrochemically polymerized films. A review. Biosensensors \& Bioelectronics. 1999; 14: 443-456.

10. De Queiroz AAA, Vargas RR, Higa OZ and Ribeiro RR. Lactam-amide graft copolymers as novel support for enzyme immobilization. Journal of Applied Polymer Science. 2002; 84(4): 767-777.

11. Bradford MM. A rapid and sensitive method for the quantitation of microgram quantities of protein utilizing the principle of protein-dye binding. Analytical Biochemistry. 1976; 72: 248-254.

12. Fernandes EGR, Soares DAW and De Queiroz AAA. Electrical properties of electrodeposited polyaniline nanotubes. Journal of Materials Science Materials in Electronics. 2008; 19(5):457-462.

13. Albery WJ, Bartlett PN, Cass AEG, Craston DH and Haggett BGD. Electrochemical sensors: theory and experiment. Journal of the Chemical Society Faraday Transactions 1. 1986; 1: 1033-1050.

14. Yang Y and Mu S. The bioelectrochemical response of the polyaniline sarcosine oxidase electrode. Journal of Electroanalytical Chemistry. 1996; 415(1-2): 71-77.

15. Yun YH, Dong Z, Shanov VN, Heineman WR, Halsalld HB, Bhattacharya A et al. Nanotube electrodes and biosensors. Nano Today. 2007; 2: 30-37.

16. Trojanowicz M. Analytical applications of carbon nanotubes: a review. TrAC Trends in Analytical Chemistry. 2006; 25(5): 480-489.

17. Guan Z. Control of Polymer Topology by Chain-Walking CatalystsChemistry- A European Journal. 2002; 8(14): 3086-3092.

18. Laskin AI. Enzymes and immobilized cells in biotechnology. EUA: Benjamin/Cummings Co; 1985.

19. Bartlett PN, Bradford VG and Whitaker RG. Enzyme electrode studies of glucose oxidase modified with a redox mediator. Talanta. 1991; 38(1):57-64.

20. Kamin RA and Wilson GS. Rotating-ring-disk enzyme electrode for biocatalysis kinetic-studies and characterization of the immobilized enzyme layer. Analytical Chemistry. 1980; 52: 1198-1205.

21. Wang Z, Liu S, Wu P and Cai C. Detection of glucose based on direct electron transfer reaction of glucose oxidase immobilized on highly ordered polyaniline nanotubes. Analytical Chemistry. 2009; 81(4): $1638-1645$.

22. Zhou H, Chen H, Luo S, Chen J, Wei W and Kuang Y. Glucose biosensor based on platinum microparticles dispersed in nano-fibrous polyaniline. Biosensensors \& Bioelectronics. 2005; 20: 1305-1311.

23. Schuhmann W, Kranz C, Wohlschlager H and Strohmeier J. Pulse Technique for the Electrochemical Deposition of Polymer films on Electrode Surfaces. Biosensensors \& Bioelectronics. 1997; 12(12):1157-1167. 